\title{
Medium-capacity transit systems: some reflections about making the right choice
}

\author{
M. Novales, A. Orro, E. Conles \& J. Anta \\ Group of Railways and Transportation Engineering, \\ University of A Coruña, Spain
}

\begin{abstract}
Medium-capacity transit systems (MCTS) cover the wide range existing between low-capacity systems (usually operating in streets with mixed traffic, with regular bus and tramways as the main solutions) and high-capacity systems (with exclusive right-of-way, as subway or suburban railways). MCTS are characterized by the use of a reserved right-of-way, which is longitudinally physically separated by curbs, barriers, grade separation and the like from other traffic, but with grade crossings for vehicles and pedestrians, including regular street intersections. The more common examples of MCTS are light rail transit (LRT) and bus with a high level of service (BHLS).

Nowadays, BHLS has proven to be able to get the same performance as LRT in the most of the fundamental subjects, leading, in general, to lower capital costs. Nevertheless, there still is a certain tendency to think about LRT as a "better" system than BHLS. This tendency is based on the fact that BHLS is still associated to regular buses operation, and that people, even technicians, are not aware of the improvements in performance that can be achieved changing the operation philosophy of bus transit systems.

In this paper, a comparison between LRT and BHLS will be made highlighting their main differences and similarities, and noting which of the assumed advantages of LRT are real and which others can be achieved by BHLS with a cared operation, taking the light rail concept to bus transit systems by the idea "think on LRT, give the service with buses".

Keywords: medium-capacity transit system, light rail, bus with high level of service, mode choice, transport planning.
\end{abstract}




\section{Introduction}

Existing solutions for metropolitan public transport can be classified with regard to its maximum transport capacity (passengers per hour), which is linked to vehicles, operation modes and infrastructure. Low-capacity systems, like bus or tram, usually share the right-of-way with private transit. High-capacity systems, like subway or commuter rail, have exclusive ways, being not accessible to any other users. Medium-capacity metropolitan transit systems (MCTS) are characterized by operating with exclusive right-of-way in the most part of their route, allowing grade crossings with other users. Most common examples of MCTS are light rail transit (LRT) and buses with high level of service (BHLS), even though there are also intermediate solutions like guided buses and rubbertyred light rail.

LRT systems have been widely popular in recent years, and this has led that in many cities this is the only option considered when a MCTS is going to be implemented. The fact that BHLS can solve the problem with similar performance and lower costs is not known or taken into account in many cases. Preconceived approaches about the greater attraction for travellers and better performance of LRT usually influence this decision, which may not be properly substantiated and must be adapted depending on the specific case of implementation.

This paper will highlight common features and differences of LRT and BHLS, trying to establish a basis for comparing fairly and objectively both systems. This is an attempt to clarify how far the advantages and disadvantages of each system are real, indicating the points where a further research is needed to draw clear conclusions. This in-depth research will be conducted in the project 'Technological, economic and user-attraction comparison of new advances in medium-capacity transit systems' that the authors are currently developing.

\section{Common characteristics of the SCI}

Medium-capacity transit systems (MCTS) are characterized by exclusive rightof-way in most of their path, that is, the urban space area used is separated from other traffic by bollards, curbs, hedges or other systems, but there are grade crossings with other users [1]. These crossings are needed because, in most cases, the MCTS run on the surface of the city streets, so it is imperative to provide continuity to the lanes that intersect their trace.

The fact of operating with exclusive right-of-way, and therefore being not influenced by urban congestion, provides the MCTS with a set of positive features. They can meet their schedules and frequencies with high reliability, providing also high commercial speeds (Table 1). Safety and ride comfort are greater as they do not share lanes/tracks with other vehicles. The highest commercial speeds result in reduced operating costs for the transit operator.

These features make the MCTS be competitive with private vehicles, especially in presence of congestion, leading to a greater attraction for potential users, including the non-captive (those who have other possible choices for 
Table 1: $\quad$ Commercial speeds of MCTS.

\begin{tabular}{|c|c|c|c|c|c|}
\hline System and city & \multicolumn{3}{|c|}{ Speed (km/h) } & Source \\
\hline \multirow{2}{*}{ LRT Madrid (Spain) } & ML1 & ML2 & ML3 & ML4 & $\begin{array}{c}\text { Consorcio Regional de } \\
\text { Transportes de Madrid (2009) }\end{array}$ \\
\cline { 2 - 4 } & 18.43 & 23 & 25 & 17 & \multirow{2}{*}{ Metro do Porto (2010) } \\
\hline \multirow{2}{*}{ LRT Porto (Portugal) } & Violet L. (min) & Red L. (max) & {$[2]$} \\
\cline { 2 - 4 } & \multicolumn{3}{|c|}{14.55} & 32.30 & {$[3]$} \\
\hline BHLS Grenoble (France) & \multicolumn{3}{|c|}{17.5} & {$[3]$} \\
\hline BHLS Rouen (France) & \multicolumn{3}{|c|}{21} & \\
\hline BHLS Nantes (France) & \multicolumn{3}{|c|}{} \\
\hline
\end{tabular}

transport). This is reinforced with a more powerful and prestigious image than systems without exclusive right-of-way.

These systems have an impact on land use that does not occur in the case of public transport with shared way, due to its characteristic of 'permanence' in time, inasmuch as the elimination or substantial modification of the service is perceived as more difficult $[4,5]$.

As can be seen, all these advantages of MCTS are derived exclusively from the reserved right-of-way, being independent of the used technology (whether the service is provided by buses, light rail or intermediate systems).

Moreover, these benefits provided by the reserved right-of-way are complemented, in many cases, by additional measures to further enhance the commercial speed of the system, as prioritization in traffic lights, adequate distance between stops [6], payment systems without driver intervention and quick access at the level of the vehicle's floor.

Although these additional measures are more common in LRT than in bus systems, there is no technical impediment to apply them to the latter, thus reaching the concept of 'bus with high level of service' (BHLS), which then becomes a road mode with similar features to those of light rail.

The final goal of all these strategies is to achieve a high commercial speed, at around $20 \mathrm{~km} / \mathrm{h}$ in the city centre and $25 \mathrm{~km} / \mathrm{h}$ in the periphery, with the aim of that public transport becomes more competitive than private vehicle [7]. As comparative value, the average speed measured by 'floating car' in the city of A Coruña (Spain) in the year 2009 was $19.49 \mathrm{~km} / \mathrm{h}$ on a working day.

\section{Differentiating characteristics between LRT and BHLS}

In this section the characteristics that are generally considered to differentiate the MCTS will be presented. As will be shown, some of these differences are clear and plausible while others (such as operating costs, the higher attraction of noncaptive users, etc.) are based on incorrect assumptions or are not sufficiently substantiated even though they are generally accepted.

\subsection{Guidance system}

Rail vehicles (of LRT) are characterized by automatic guidance, caused by the contact between wheel and rail. Rubber-tyred light rail uses a guidance system quite similar. 
Generally buses are guided through steering wheel by means of the friction between wheel and road. This is still the standard technology, although in recent decades there have been developed different technologies of automatic guidance for buses, as in the case of guidance through horizontal wheel against vertical surface (used, for example, in Essen), or optical guidance in Rouen. In the following paragraphs the implications of conventional guidance versus rail guidance are analyzed, as well as the ways to mitigate these differences.

On buses without automatic guidance the vehicle's trajectory is entrusted to the driver's skill. This results in less comfort for users and a larger crosssectional space occupation for a certain width of the vehicle. This becomes less important as bus vehicles tend to be somewhat narrower than light rail ones. Moreover, in the case of LRT, the space occupation of the supporting posts of the overhead line has to be also considered, so the total space occupied by the two types of systems is quite similar. The difference in comfort can be reduced by using suitable vehicles and training drivers to control lateral accelerations.

The obtainment of a proper positioning of the vehicle at stops is more difficult, so that horizontal gap between the platform and the vehicle tend to be larger, hindering the quick and easy access of users, especially for disabled people. This drawback can be overcome if stops are arranged in areas with proper layout and with a successful design of the platform. The solution with access ramps is complex because it can increase the time spent at the stop.

In case of breakdowns, accidents or other incidents, the bus is able to leave its own lanes to access other areas of the street shared with other modes. This feature is, in this case, a competitive advantage compared with systems that can run only on areas where the guidance elements are installed, which makes them more rigid to face these kinds of exceptional circumstances.

\subsection{Size of vehicles, service frequencies and maximum capacities}

Generally BHLS vehicles are smaller and with lower capacity than LRT. Articulated buses are typically $2.55 \mathrm{~m}$ wide with a maximum length of $18 \mathrm{~m}$, which give rise to a maximum capacity of around 100 passengers/vehicle, assuming density equal to 3 passenger $/ \mathrm{m}^{2}$, which is considered an appropriate value to ensure adequate comfort for the users of this mode [6]. Bi-articulated buses, with maximum lengths around $25 \mathrm{~m}$, have a maximum capacity of about 150 passengers/vehicle, although they are very unusual.

Most common LRT vehicles are between 30 and $45 \mathrm{~m}$ long, with capacities of 165 and $250 \mathrm{p} / \mathrm{veh}$, respectively, assuming density of $3.5 \mathrm{p} / \mathrm{m}^{2}$. They can form LRT-trains typically composed by two vehicles and rarely by three. The maximum length of the LRT-trains is regulated differently in each country: $60 \mathrm{~m}$ in the UK, $75 \mathrm{~m}$ in Germany and $120 \mathrm{~m}$ in United States [8]. This means that LRT-trains have capacities up to 330 or even $500 \mathrm{p} /$ train.

If a frequency of 5 minutes between units is considered, the capacity of a BHLS would range, with the data above, from 1200 passengers/hour (articulated) to $1800 \mathrm{p} / \mathrm{h}$ (bi-articulated), while capacity of LRT would be ranged from $1980 \mathrm{p} / \mathrm{h}(30 \mathrm{~m})$ to $3000 \mathrm{p} / \mathrm{h}(45 \mathrm{~m})$ with single vehicles (up to $6000 \mathrm{p} / \mathrm{h}$ with double-units of $90 \mathrm{~m}$ ). Therefore, LRT would reveal as the most appropriate 
solution for high demand corridors. In many cases the demand is not as high as these, so the capacity would not be a proper justification for the choice of LRT.

In the LRT of Madrid (Spain), ML 1 was the line with the highest hourly demand in 2009, with a maximum of 1662 passengers per peak hour and direction (ppphpd). This demand could be met by a bus system with frequency slightly below 4 minutes. One of the LRT with greatest demand in Spain is Tenerife, with a maximum demand of 2619 ppphpd. Of course, BHLS systems can reach much higher values of capacity, depending on the level of right-of-way and management, as shown the 34595 ppphpd moving in the South corridor of the Curitiba's network (Brazil) [9].

If values corresponding to the articulated vehicle are considered as typical reference for buses, and those of the $45 \mathrm{~m}$ long single unit for LRT, the latter reaches a peak hour capacity per direction by 2.5 times higher for the same frequency of service. Alternatively, the same demand could be met with a timeinterval between vehicles 2.5 times higher, which would result in a lower amount of vehicles and drivers needed, and therefore, lower operating costs. However, it should be noted that frequency is one of the most valued features by users to characterize system's quality, and it plays an important role in attracting travellers. In this regard, one should remember that weight of waiting time is 2 or 3 times the weight of time spent in the vehicle when the generalized cost of travelling between two points is calculated [10].

\subsection{Ability to attract passengers}

In many cases the argued reason for the implementation of LRT instead of BHLS is based on the perception that the latter will be less attractive to people. It is widely quoted the sentence, attributed to a politician of Los Angeles, "Trains are sexy, buses are not" to justify that LRT systems have an ability to attract users much stronger than a buses-based system with similar services. This had often led to have chosen expensive systems, fact that should be reviewed in the current economic context, given that this higher attractiveness is controversial and that local circumstances and perceptions should be also considered.

From the point of view of the most renowned specialists in travel behaviour modelling, this statement is not sufficiently supported by the experiments performed. David Hensher (Lifetime Achievement Award from the International Association of Travel Behavior Research, 2009) thinks that there is not anything inherently more attractive in LRT than in the bus, asserting that investments in improving bus systems, with reservation of right-of-way, prioritization and travel times similar to the LRT, are equal and often more effective in attracting travellers, but with lower costs [11]. In Ben-Akiva and Morikawa [12] this problem is discussed by means of revealed and stated preference data, concluding that there is not a preference for rail transport in the cases studied, but further research would be needed on this field. Lillo et al. [10] state that a rail system (strictly on a fixed support of any kind) has an intrinsic advantage between 2 and 15 generalized minutes on conventional bus (considering the generalized cost of travelling between two points as the weighted sum of travel time, waiting time, access time and monetary costs) but that a well-designed and 
implemented BHLS system can be almost as well-rated by the users as a tram or a LRT, assigning to BHLS a modal advantage of about 7 to 10 minutes if compared with conventional bus services.

Other authors, such as James [5], ensure that compared with LRT, a bus with exclusive right-of-way is not able to attract as many non-captive users. Similarly, in a manual of a public organization [13] is believed that many users prefer the LRT rather than bus, even when there are no differences in travel times or prices, and it is added that this additional attraction of rail systems may be due to factors such as: rail systems cannot easily change, so the users can know and understand the system easily; the permanence of these systems makes people plan their lives around them with confidence, making the choice of transport based on the availability of these; this permanence also enhances the establishment of businesses along the routes, which concentrates development in a way that it can be served more efficiently by public transport; and the system can be viewed and advertised by itself.

Finally, Scherer [14] states that LRT has higher ability to attract travellers and tries to determine the characteristics that make it more attractive than a BHLS with the same quality of service. This article approaches the problem from a cognitive point of view, indicating that an important factor in customer choice may be the visibility of the system. Among the factors with influence on the visibility of a public transport system she highlights four groups: 1) Newness: new transport system implementation, new vehicles, new real-time information systems and service to new destinations; 2) Visual design: special design for the vehicles, design of stops and use of bright colours; 3) Visual dominance: visibility of routes due to the tracks or dedicated lanes, visibility of stops and visual dominance due to high frequencies; 4) Presence: strong presence in the media, image enhanced by the media and public debate on transportation projects.

As can be seen, LRT has, in general, more visibility attributes, and it is also very easy to identify the lines due to the presence of the rails and the overhead line. However, most of the visibility attributes could be transferred quite efficiently to bus systems, for example by painting the dedicated lanes in a different colour, by using vehicles with a different look, by enhancing the debate in the media about new features provided to the system, etc.

Therefore, as has been shown in the preceding paragraphs, the greater ability for attracting users of LRT compared with BHLS is a controversial issue that requires the completion of a detailed study to clarify it. On the other hand, if this advantage is proven to be real, it would be interesting to determine the characteristics of LRT that result in this increased attraction, to see how far they could be transferred to bus systems.

\subsection{Implementation costs}

Construction of infrastructure for LRT systems is generally more expensive than for BHLS, as the latter enables the avoidance of very costly works such as the rail tracks and the electric power line (except trolleybus) and, additionally, 
vehicles depots and maintenance facilities are less complex. However, some subtle distinctions should be taken into account in this regard.

The more similar to LRT the performance, reliability and image of a BHLS system are intended to be achieved (i.e. not only providing right of way), the nearer to LRT the required investment for BHLS will need to be (although, in general, the latter will not become equal to the first one). The diversion of urban services pipelines in order to avoid later disturbances able to reduce the system's reliability is one of the possible investments (this activity usually amounts about $10 \%$ of the investment in LRT implementation depending on the percentage of on-street running [13]). Furthermore, stops/stations, vehicles and corridors where they run should be carefully arranged with the aim of achieving a strong image for the system. Moreover, it must be provided with passenger information devices, traffic priority systems, etc. similar to those used in LRT, thus entailing the respective costs.

The implementation of LRT systems usually implies renewals in the cross section of the affected streets (with improvements in urban furniture, pavements, etc.), which entails additional costs. Nonetheless, these costs would be common to BHLS if this system aims to have comparable urban effects.

The costs of infrastructure investment for LRT systems are usually ranging from 15 to 50 million euros $(\mathrm{M} € / \mathrm{km})$, while these costs are comprised between 1 and $10 \mathrm{M} € / \mathrm{km}$ [10] for BHLS. In the case of LRT, the highest values are generally related to larger stretches of independent separate way (underground or elevated) and to a stronger effort in urban regeneration. For BHLS, the higher costs are linked to cases where a new roadway was specifically constructed for the system (table 2).

Table 2: $\quad$ Construction costs for MCTS.

\begin{tabular}{|c|c|c|c|}
\hline & Line and city & $\mathrm{M} € / \mathrm{km}$ & Comments and sources \\
\hline \multirow{6}{*}{ 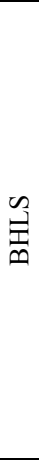 } & $\begin{array}{l}\text { Transmilenio. Bogotá } \\
\text { (Colombia) }\end{array}$ & 5.3 & $\begin{array}{l}\text { Infrastructure, including stations, pedestrian bridges } \\
\text { and control centre [10] }\end{array}$ \\
\hline & $\begin{array}{l}\text { Key Route Bus System. } \\
\text { Nagoya (Japan) }\end{array}$ & 2 & $\begin{array}{c}\text { Dedicated lane with right of way and some stretches of } \\
\text { independent track at elevated level. Axles guidance } \\
\text { like in the O-Bahn (Essen) [2] }\end{array}$ \\
\hline & $\begin{array}{c}\text { Trunk Network. } \\
\text { Stockholm (Sweden) }\end{array}$ & 1 & Infrastructure without vehicles: $0.6 \mathrm{M} € / \mathrm{km}[2,15]$ \\
\hline & TEOR. Rouen (France) & 5.5 & $\begin{array}{c}\text { Guidance system by means of camera in stops and } \\
\text { universal accessibility [2] }\end{array}$ \\
\hline & $\begin{array}{l}\text { Busway. Nantes } \\
\text { (France) }\end{array}$ & 7 & $\begin{array}{l}\text { Including projects and infrastructure, but without } \\
\text { vehicles }\end{array}$ \\
\hline & $\begin{array}{l}\text { TVRcas, TRAM. } \\
\text { Castellón (Spain) }\end{array}$ & 9.8 & $\begin{array}{l}\text { With trolley (overhead line for electric power), optical } \\
\text { guidance all over the entire length and roadway } \\
\text { painted in red [3] }\end{array}$ \\
\hline \multirow{4}{*}{ 卢 } & Parla (Spain) & 14.1 & \\
\hline & Murcia (Spain) & 14.6 & {$[16$, article on 13/07/2009 ref. 3911] } \\
\hline & Porto (Portugal) & 19.3 & $\begin{array}{c}\text { Line } F, 7 \mathrm{~km} \text { length of which approx. } 1 \mathrm{~km} \text { runs in } \\
\text { tunnel [16, article on 14/01/2011 ref. 6524] }\end{array}$ \\
\hline & Tenerife (Spain) & 23.9 & Line 2 [16, article on 29/05/2009 ref. 3651] \\
\hline
\end{tabular}


As regards the cost of vehicle purchase, the price of a standard articulated bus is, in average terms, about $0.35 \mathrm{M} €$, while the acquisition cost of a LRT vehicle is around 2.5 M€ [1]. However, the longer service life of LRT vehicles (on the order of 25-30 years versus 8-10 years for buses) and their greater capacity (able to reduce the required amount of vehicles, although this is sometimes constrained by the service frequency). The purchase cost of BHLS vehicles is subjected to considerable increases if they incorporate guidance systems or propulsion engines different from diesel motor with the aim to reduce emissions and improve the energy efficiency.

\subsection{Operating cost}

In general terms, the larger capacity of LRT units (single or coupled) implies lower operative costs in case of high hourly demand. Nevertheless, there are many time intervals in which this greater capacity will not be actually exploited inasmuch as certain minimum frequencies must be preserved. Some studies point out that if the effective use of the vehicles (demand) is analyzed instead of the capacity of the line (i.e. the cost per used place instead of per offered place), LRT systems lead to higher costs than buses with dedicated lanes. According to Hensher [17], the most cost-effective LRT would give rise to greater unit operating costs than its road-based opponent by $60-80 \%$. However, there are some authors who assert the contrary, as can be seen in James [5].

Concerning the maintenance costs, controversies are also found among the diverse authors. Some of them affirm that these costs are higher for bus systems because of the greater costs consumed in roads maintenance and the faster deterioration of the vehicles, which has to be replaced sooner [5]. On the contrary, some references argue that buses with dedicated lanes/roadways are easier to operate and maintain than LRT systems because the latter usually require complementary systems with much more complex devices for communication, signalling, propulsion and control [17]. Nevertheless, it should be kept in mind that the more the aimed performance of the road-based systems is close to that of the rail-based ones, the more similar the complementary systems (communication, management, etc.) will need to be.

Therefore, as can be seen, the difference of costs between LRT and BHLS systems may be significant, and it is not possible to asseverate in advance whether the higher implementation costs will be outweighed by the lower operating costs (this question will be determined by the demand of the transit system). Thus, it would be interesting to ascertain whether the greater cost of LRT systems is compensated by a bigger social benefit, which will justify the investment, or, on the contrary, it does not worth this investment effort. Clearly, the answer to this question depends on the circumstances of each particular case, so the conditions able to tip the balance in favour of one or the other system should be determined.

\subsection{Impacts on land use}

The effects of the guided systems on land use are generally more intense than in the case of systems based on conventional buses, since certain guarantee of 
permanence is often considered as necessary by the private sector to invest in urban developments conditioned by the public transit services of the area. This guarantee of permanence is much weaker for the services based on dedicated lanes for buses than in the case of rail-based systems, whose investment is quite higher and, therefore, they are less 'reversible'. Nevertheless, these guarantees should be obtainable through long-term political agreements intended to avoid that a change of governing party in a city council results in significant modifications in the planning of the metropolitan transport systems, as these sharp turns usually entail a sequence of inefficient actions and thus the waste of public funds without having ensured good quality services for citizens.

Anyway, it would be interesting to study further the influences that the implementation of guided road-based MCTS has caused on land use.

\subsection{Layout and capability for urban insertion}

Bus-based systems are able to run on more constrained geometries than LRT (minimum turn radius of $11 \mathrm{~m}$ for buses versus $15 \mathrm{~m}$ for LRT) and without special limits in maximum longitudinal gradient, which is about $85 \%$ for LRT (see case of Tenerife). More data about the layout geometry of LRT systems can be found in [18]. However, these capabilities of BHLS should not be fully exploited in order to not penalize excessively the average operating speeds and the passengers' ride comfort. One of the general recommendations in order to upgrade a bus system with dedicated lanes to the status of BHLS is to be careful in the geometric design of the layout (for instance, by making the bus lane cross the roundabouts through the central island, similarly to most LRT systems). Therefore, it would be concluded that the insertion into the urban layout does not constitutes a distinctive or significant feature for the choice of a certain MCTS.

In the case of LRT, it is a usual practice that these systems transit pedestrian streets in the town centre, where their speed is limited to a maximum of $25 \mathrm{~km} / \mathrm{h}$. As LRT is a guided system, it is possible to mark the clearance area on the pavement in consideration of the vehicle motions with the aim to prevent risk situations for pedestrians. On the contrary, the transit of buses through pedestrian zones is not a common practice (especially if they are articulated) since the taken risk would be greater. This factor can be interpreted as a disadvantage of the busbased systems in certain corridors. However, in many occasions the pedestrianizations are implemented simultaneously to the introduction of the new MCTS, in which case the strategy for bus systems would consist in a differentiated treatment of the street, with removal of private vehicles and preservation of a roadway or lane for buses and sidewalks for pedestrians.

\subsection{Environmental impact and energy efficiency}

LRT vehicles, given that they are electric-powered, do not produce emissions in the zones where they travel (but the generation of electric energy may sometimes give rise to emissions in the production areas). Furthermore, LRT vehicles provided with energy storage systems mounted on-board (generally by means of supercapacitors) are becoming increasingly common nowadays. These systems 
enable the recovery and storage of kinetic energy under braking and its later use under acceleration, which can lead to energy savings by up to $30 \%$.

On the other hand, electric engines are more silent than internal combustions ones, so the acoustic pollution also decreases. However, it should be taken into account that LRT systems could lead to problems concerning the transmission of vibration to the surroundings of the tracks, so additional measures would be sometimes necessary to avoid this kind of disturbance, chiefly if the nearest environment is especially sensitive.

Nevertheless, the environmental vantage of LRT can be neutralized by the BHLS systems if certain technological advances are introduced, such as vehicles propelled by natural gas engines or the use of fuel cells or other clean and efficient power systems. Furthermore, the employment of hybrid buses (electric+diesel) and buses with supercapacitors is able to allow the use of strategies for energy recovery similar to those of the LRT. Other possibility is the employment of vehicles of trolleybus type (case of Castellón), which are provided with electric engine powered through overhead line. There are some other technologies in which the power supply takes place through an electrical conductor embedded into the pavement, which is activated by means of a capture device attached to the bottom of the vehicle. In these cases, some clarifications should be taken into account concerning the system flexibility and other characteristics pointed out in this paper. The increase of cost entailed by the use of power systems different from diesel engines must be borne in mind.

\subsection{Flexibility in operation}

Flexibility in operation is usually argued as one of the main advantages of the BHLS systems in relation to LRT, since the latter can only run on specially purposed tracks. Even the guided buses are able to run, by means of conventional driving, on stretches in which the guidance mechanism is not available.

This feature can result in an important competitive advantage, mainly in case of anomalous operating conditions, as the buses are able to leave their own roadway/lane in the stretch where the incident has taken place and thus keep on providing service. In a similar circumstance, a LRT system would be much more compromised inasmuch as, in the most unfavourable case, the vehicles could not bypass the obstructed section and the service would have to be suspended, at least in certain stretches. In a more propitious case, the operation could be continued by diverting the traffics from double track to single shared-track between two crossovers, but with significant drawbacks for the capacity and reliability of the system.

Furthermore, the flexibility of buses enables the possibilities of spreading the services towards peripheral areas of lower density by making the bus run on shared lanes/roadways through zones without significant traffic congestion. This operation mode provides direct services with high quality and competitive average operating speeds and, moreover, avoids the need for transhipments, which imply a waste of time and are negatively perceived by the users. Nevertheless, such flexibility could become the worst enemy of a bus system with dedicated right-of-way if it is not properly managed. For instance, if it is 
intended that this system reaches all possible origins and destinations, this aim can lead to tortuous and excessively long routes, with too many stops and travel times too high, thus resulting in a worsening of the service quality. Another example could consist in changes in the operation patterns, such as the conversion of certain stretches of dedicated lane into shared lanes, frequent changes in the routes, etc., which can lead to a loss in quality affecting the ability for attracting users.

In short, the flexibility of the bus-based systems can be considered as an advantage, but it should be handled with some care to achieve and conserve the status of BHLS and thus be not only a bus with dedicated right-of-way.

\section{Conclusions}

Both bus with high level of service (BHLS) and light rail transit (LRT) are transport technologies suitable for satisfying intermediate demands, with advantages based essentially on the employment of reserved right-of-way. The choice of which technology is the most favourable to be implemented in a specific case needs a detailed analysis of the particular circumstances. The comparison between one and other system is not simple: there are some controversial issues and others either not sufficiently known by the decisionmakers or requiring more in-depth research.

In general terms BHLS systems can be considered able to achieve, partially or completely, many of the positive features inherent to LRT systems - such as comfort of guidance, energy efficiency or effects on land use - if adequate measures have been taken in the planning, design, operation and marketing of the BHLS services. Nevertheless, these measures reduce the more favourable features of BHLS systems as regards cost and flexibility of routes and operation. The transport capacity per unit of the LRT systems is higher, but in many cases this greater capacity is unnecessary for fulfilling the demand or it would lead to unfavourable frequencies for attracting potential passengers.

The inherent attractiveness of the systems is the topic that generates greatest differences of opinion. The general perception, informative publications [13] or young researchers $[5,14]$ hold that light rail is considerably more attractive by itself. Nonetheless, the most renowned world experts in study of travel behaviour disagree with this statement $[10-12,17]$, reflecting in general the need for further research on this field.

\section{Acknowledgements}

The research project 'Technological, economic and user-attraction comparison of new advances in medium-capacity transit systems (NASCI)', on which the elaboration of this paper is based, is funded by the Ministry of Science and Innovation within the framework of the R\&D\&I National Plan 2008-2011. Additionally, the authors acknowledge the collaboration from Tomás Melero ('Consorcio Regional de Transportes de Madrid'), Pedro Costa ('Metro do Porto'), Joan Carsi ('TRAM de Barcelona') and Andrés Muñoz de Dios 
('Metropolitano de Tenerife') for having supplied interesting data about their respective transit systems.

\section{References}

[1] Novales, M. \& Bugarín, M.R. Tranvitrén y tren-tranvía. Hacia una mejora del aprovechamiento de las infraestructuras ferroviarias. Colegio de Ingenieros de Caminos, Canales y Puertos: Madrid, 2009.

[2] CERTU. Bus à Haut Niveau de Service. Concept et recommandations. Ministère des Transports de l'Équipement du Tourisme et de la Mer. 2005.

[3] Valdés, C. \& López, M.E. Del BRT al BHLS: un enfoque europeo de los sistemas de transporte masivo en autobús. Actas del IX Congreso de Ingeniería del Transporte, CIT 2010. Madrid, 2010.

[4] Vuchic, V.R. Urban Transit Systems and Technology. John Wiley \& Sons Inc.: New York, 2007.

[5] James, D. Ottawa's Transitway: From Busway to Light Rail. Master's Degree Project. University of Calgary, 2008.

[6] Cristóbal, C. Reflexión sobre los modos de transporte público en las ciudades. Ingeniería y Territorio, 86, pp. 32-39, 2009.

[7] Andersson, P.-G., Gibrand, M. \& Kottenhoff, K. Bus Rapid Transit in Sweden? KTH: Stockholm, 2009.

[8] Union International des Transports Publics (UITP). Guidelines for Selecting and Planning a New Light Rail System. UITP: Brussels, 2004.

[9] Cristóbal, C. \& González, J.D. (eds). Buenas prácticas de Transporte Público en Europa y América Latina: Conclusiones del proyecto europeo PROMOTEO. Consorcio Regional de Transportes de Madrid, 2007.

[10] Lillo, E., Wensell, U. \& Willumsen, L. Innovación en el transporte urbano: Bus Transit Systems. Economía Industrial 353, pp. 65 - 72, 2003.

[11] Hensher, D. A. Urban public transport challenges. The Drawing Board: An Australian Review of Public Affairs, 1 (2), pp. 47-62, 2000.

[12] Ben-Akiva, M. \& Morikawa, T. Comparing ridership attraction of rail and bus. Transport Policy, 9 (2), April 2002, pp. 107-116.

[13] Green light for light rail. Department for Transport, 2011. Online: assets.dft.gov.uk/publications/light-rail/green-light-for-light-rail.pdf

[14] Scherer, M. Is light rail more attractive to users than bus transit? Transportation Research Record, 2144, pp. 11-19, 2010.

[15] Sedin, S. SES in central Stockholm. Online: www.bhls.eu/IMG/pdf /Trunk Network assement report 1993.pdf

[16] Vía Libre. La Revista del Ferrocarril, www.vialibre-ffe.com

[17] Hensher, D. A. A bus-based transitway or light rail? Continuing the saga on choice versus blind commitment. Road \& Transport Research, 8 (3), pp. 320, 1999.

[18] Novales, M., Orro, A. \& Bugarín, M.R. Track geometry for light rail systems. Transportation Research Record, 2146, pp. 18-25, 2010. 